\title{
Gestational Timing of Prenatal Disturbance and Fetal Sex Determine the Developmental Outcomes
}

\author{
Danielle N. Rendina Gabriele R. Lubach Christopher L. Coe \\ Harlow Center for Biological Psychology, University of Wisconsin, Madison, Wis., USA
}

\section{Key Words \\ Infant development · Prenatal stress · Cortisol · Prenatal programming $\cdot$ Temperament regulation $\cdot$ Neuromotor development $\cdot$ Rhesus monkey $\cdot$ Macaca mulatta}

\begin{abstract}
Background: Maternal stress during pregnancy can have deleterious consequences, increasing risk for prematurity and low birth weight, as well as postnatal effects on emotional regulation and neuromotor development. It is less clear, however, whether moderate and brief gestational disturbances have similar effects. Objective: To determine the impact of a delimited period of moderate maternal stress on infant growth, emotional reactivity, and neurobehavioral maturity in a nonhuman primate model. Methods: Eightythree infant rhesus monkeys were generated from disturbed pregnancies, either early or late gestation, and compared with 51 undisturbed infants. Maternal stress was induced with an acoustical startle protocol for $25 \%$ of gestation. Infant weights, anthropometrics, and neurobehavioral data were obtained. Analyses focused on differential effects of prenatal stress on male and female infants. Results: The disturbance manipulation elevated cortisol levels acutely in the gravid females and they gained less weight by term. Never-
\end{abstract}

theless, female infants from the early stress condition were significantly larger at birth. This differential growth trajectory was then sustained through 6 months of age. Infants from stress conditions were more emotionally reactive and evinced immature neuromotor reflexes, especially when gestated by late stress mothers. Conclusions: Even moderate maternal disturbance impacted infant temperament and neuromotor development in this nonhuman primate model. Effects on fetal and infant growth differed from typical reports of growth inhibition, both in other animal species and human studies. The findings convey the importance of considering the duration and severity of prenatal insults, and the potential for fetal plasticity and recovery, permitting compensatory growth responses.

๑) 2016 S. Karger AG, Basel

\section{Introduction}

Clinical research and studies in animals have demonstrated that the emotional and physical state of a pregnant female can have both immediate and enduring effects on infant development. When severe and sustained, prenatal stress increases the risk for premature birth and low birth weight, and has also been correlated with a predisposition

\section{KARGER}

E-Mail karger@karger.com

www.karger.com/neo (c) 2016 S. Karger AG, Basel

$1661-7800 / 16 / 1094-0314 \$ 39.50 / 0$
Danielle N. Rendina, MS

Harlow Center for Biological Psychology, University of Wisconsin

22. N Charter Street

Madison, WI 53715 (USA)

E-Mail rendina@wisc.edu 
for later health concerns, including obesity, poor glucoregulation, and cardiovascular disease [1]. In addition, maternal stress in animals can affect neurobehavioral and motor development and increase stress reactivity $[2,3]$. However, a close review of this literature indicates the type of disturbance, including severity and timing, influences the extent of the impact - issues addressed further in the following study.

Prenatal stress research in humans has often focused on extremely stressful events, including natural disasters, catastrophic incidents, and domestic violence $[4,5]$. By necessity, many analyses are opportunistic and retrospective, and it is not possible to control the onset and intensity with respect to gestational stage. Therefore, further investigation is still needed on more moderate maternal disturbance, which may occur during just one discrete stage of pregnancy. Studies on nutritional deprivation and teratogen exposure have indicated the effects are different depending on whether they occurred during early or late gestation $[6,7]$. In addition, when fetal stress is discrete, there is some capacity for compensatory adjustments and recovery when restricted just to early or late gestation $[8,9]$. The following experiment was designed to compare the developmental effects of moderate stress during either early or late pregnancy, both of which were followed by a period without disturbance during the remaining weeks of pregnancy.

Another area requiring further resolution is whether the sex of the fetus influences the vulnerability to prenatal disturbance. The extant literature tends to highlight the differential susceptibility of male infants $[10,11]$, but this conclusion is dependent upon the type of prenatal insult and the outcome measures. In addition, findings are likely to be different in litter-bearing species that gestate many male and female siblings simultaneously [12]. Thus, a second aim was to take advantage of the singleton pregnancies characteristic of rhesus monkeys in order to investigate differential effects on males and females.

Female rhesus monkeys were exposed to controlled stress lasting 5-6 weeks, either early or late during their 24 -week pregnancy. The immediate impact was assessed by determining maternal cortisol levels at the end of the daily manipulations. Activation of the pituitary-adrenal axis, and placental transfer of maternal cortisol, is the endocrine pathway commonly implicated as a mediator of prenatal stress, although it is known that other metabolic, placental, and growth processes can be affected [13]. Our analyses then focused on infant growth trajectories and also on their behavioral and emotional state at birth.

\section{Methods}

\section{Subjects}

Male and female infants $(\mathrm{n}=134)$ were generated from multiparous rhesus monkeys (Macaca mulatta). The adult females were healthy and between 5 and 18 years of age (mean age: $11.3 \pm$ 3.1) from an established breeding colony.

Statement of Ethics

Animal husbandry and experimental procedures were approved by the institutional Animal Care and Use Committee.

\section{Husbandry}

Females were mated with a single male for a 4- to 7-day period to verify paternity and date of conception. They were then returned to their home cages and assigned to gestational conditions. If assigned to the disturbance condition, they were relocated acutely on a daily basis for 5-6 weeks of their 24-week pregnancy, but then only minimally disturbed for cage cleaning and one blood sampling session 2 weeks before term.

\section{Prenatal Manipulations}

Eighty-three females were assigned to early and late disturbance conditions (34 and 49, respectively). An acoustical startle paradigm was used, and a moderate stress response verified by obtaining blood for cortisol determinations at 2-week intervals during the disturbance period. Females were briefly relocated daily between 14:30 and 16:00 to a darkened room. Three 110-dB sounds were broadcast for $1 \mathrm{~s}$ at random intervals during the 10min protocol. It was repeated 5 times per week during either early or late gestation (1.6-3.0 or 4.0-5.1 months after conception). Fifty-one infants ( 21 females, 30 males) served as undisturbed controls. The mothers were not manipulated other than to measure their basal cortisol levels.

\section{Maternal Variables}

Maternal weight was determined prior to conception, bimonthly during pregnancy, and at delivery to evaluate if gestational disturbance affected maternal weight gain or was related to infant birth weight.

Blood samples were assayed for cortisol levels prior to the stress manipulation, and at 2-week intervals at the end of the disturbance. Control females were sampled at the same 2-week intervals, either during early or late gestation. Blood $(1 \mathrm{ml})$ was collected quickly by saphenous venipuncture, and plasma was frozen at $-60^{\circ} \mathrm{C}$ until analyzed by iodinated radioimmunoassay (Incstar, Stillwater, Minn., USA).

\section{Infant Growth}

Infants were weighed at $1-3$ days after birth (mean $1.3 \pm 1.0$ ), and then at 2 weeks and 2, 4, and 6 months of age. All infants were full-term, natural deliveries, and weighed within the normal range $(490.6 \pm 59.6 \mathrm{~g})$. Crown-rump length, measured from the top of the head to the rump, was used to calculate BMI. Infant growth was indexed by weight, as well as rate of growth, measured as the slope of weight gain between birth and 6 months.

\section{Behavioral Analyses}

For 119 infants (77 disturbed and 42 undisturbed pregnancies), emotionality and neuromotor maturity were determined at 
2 weeks postpartum with the Infant Behavioral Assessment Scale (IBAS) [14]. A total of 49 neuromotor reflexes, attention to visual cues and sounds, responses to vestibular and tactile stimulation, and emotionality and arousal were scored. Data from this 15-min test battery were combined into four composite subscales based on a previous factor analysis of 29 informative items [15]. The four subscales were: (1) temperament regulation, (2) motor maturity, (3) sensory sensitivity, and (4) orientation. Temperament regulation captured emotional reactivity and state regulation during the test, with lower scores indicating irritability and difficult to console. Motor maturity assessed muscle tone, coordination, balance, and spontaneous movement through normative reflexes, motor activity, and speed of responding. Sensory sensitivity captured reactions to vestibular and tactile stimulation, while orientation assessed tracking of visual and auditory stimuli. Low scores on these subscales indicate immature or impaired responses.

\section{Data Analysis}

Statistical tests were conducted with the Statistical Package for the Social Sciences, Version 21.0 (SPSS) and R (R Core Team, 2015). P-P plots were used to check for skew, and nonnormally distributed variables were log-transformed to stabilize variances. Analysis of variance was used to compare the three prenatal conditions with infant sex as a between factor when appropriate. When sex was not significant, it was not included in the final analysis. In case of significant main effects or interactions, a post hoc analysis was employed to identify significant differences. Exploratory post hoc testing was conducted for one interaction of pregnancy condition and infant sex when the $\mathrm{p}$ value was $<0.06$. Repeated measure tests were adjusted with Fisher's protected LSD, and relationships between variables were assessed with the Pearson test.

\section{Results}

\section{Pregnancy Conditions}

The disturbance manipulation resulted in significantly higher cortisol levels in pregnant females, both during the early and late stress periods, as compared to the undisturbed controls $[\mathrm{F}(2,131)=41.45, \mathrm{p}<0.01]$. Cortisol in the stressed females was also acutely elevated at the end of the startle protocol as compared to their prior baseline levels (fig. 1). While similarly elevated in both periods, post hoc tests indicated the mean increment was larger during early gestation as compared to late gestation $(\mathrm{p}<$ 0.02 ). Maternal cortisol was not influenced by the sex of the fetus.

\section{Maternal Weight, Gestational Weight Gain, and Gestation Length}

Preconception weight did not vary by pregnancy condition in keeping with the random assignment. However, mothers who became pregnant with female fetuses were slightly larger $(0.3 \mathrm{~kg})$ than those who bore males, a difference that reached statistical significance $[F(1,127)=$

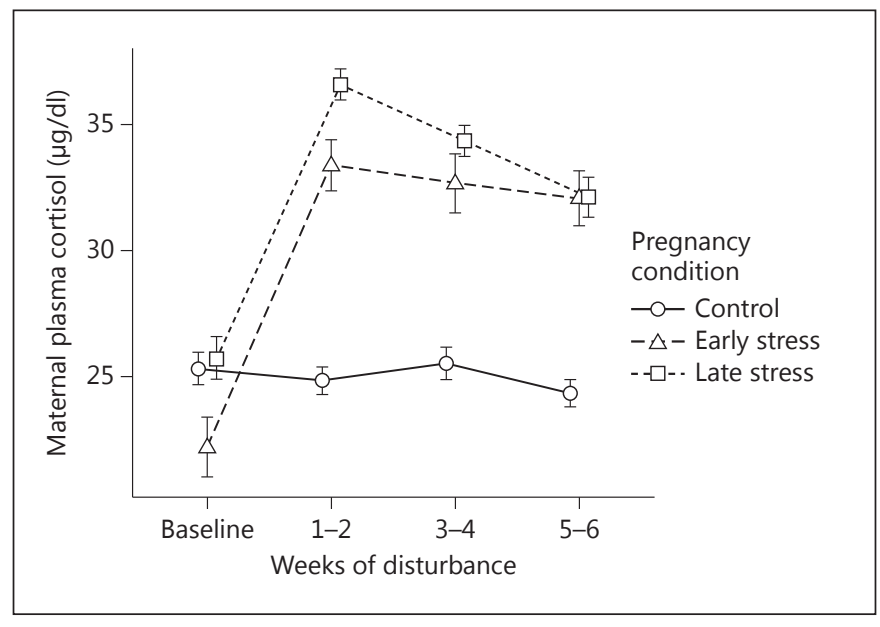

Fig. 1. Maternal cortisol response to acute disturbance during early and late gestation. Stress manipulations significantly increased cortisol from baseline levels. Cortisol levels were significantly higher in the stressed females as compared to undisturbed control females sampled at identical 2-week intervals.

4.23, $\mathrm{p}<0.05]$. Females gained a mean $1.8 \mathrm{~kg}$ across pregnancy, (95\% CI: 1.65-1.92). After accounting for preconception weight, the magnitude of the weight gain reflected an influence of the pregnancy manipulations and sex of fetus $[\mathrm{F}(2,127)=5.10, \mathrm{p}<0.01]$. The lowest maternal weight gains were in the early stress condition for mothers carrying a female fetus. On average, they gained 0.62 $\mathrm{kg}$ less than the undisturbed controls and $0.24 \mathrm{~kg}$ less than mothers in the late gestation condition with a female fetus. Overall, the inhibitory effect of the stress manipulation on weight gain was more evident when gestating a female infant than for mothers of males (table 1).

Mean pregnancy length was 168 days (95\% CI: $167.2-$ 168.8), with male infants gestating an average 1.8 days longer. Early stress condition infants gestated 2 days longer than late stress infants. However, there was not a main effect of pregnancy condition when also considering infant $\operatorname{sex}[F(2,130)=2.41, p=0.09$; table 1$]$.

\section{Birth Weight and Growth}

Prenatal stress affected the birth weight and growth of female infants. At birth, the statistical interaction between pregnancy condition and infant sex reached significance $[\mathrm{F}(2,128)=3.08, \mathrm{p}=0.05$; fig. 2a]. On average, female infants from the early stress pregnancies were $47 \mathrm{~g}$ larger than undisturbed control females and $50 \mathrm{~g}$ larger than female infants from the late stress condition. Signs of this interaction between pregnancy condition 
Fig. 2. a Female infants from the early stress condition were significantly larger at birth than females from both the control and late stress conditions $(\mathrm{p}<0.01$ and $\mathrm{p}<$ 0.01 , respectively). b At 6 months of age, female infants from the early stress condition continued to be significantly larger than females from both control and late stress pregnancies $(\mathrm{p}<0.05$ and $\mathrm{p}<0.01$, respectively). The early stress condition contained 17 males and 17 females; the late stress condition contained 19 males and 28 females. Mean \pm SEM values are portrayed. * $\mathrm{p}<0.05$ difference between pregnancy conditions.

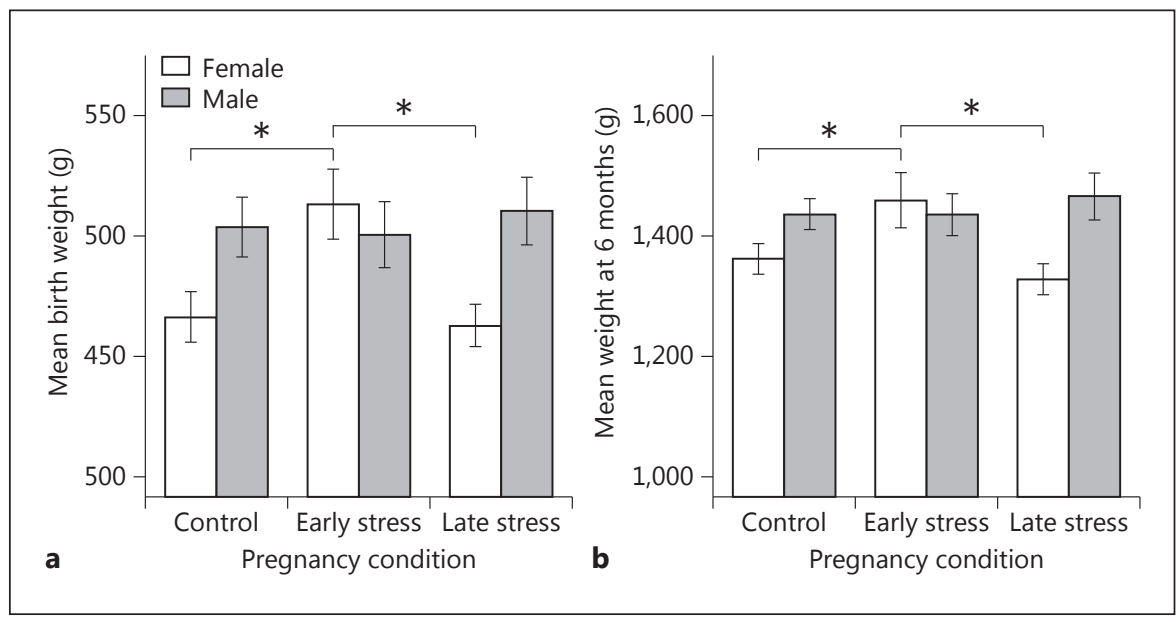

Table 1. Maternal pregnancy and infant growth parameters

\begin{tabular}{|c|c|c|c|c|c|}
\hline Maternal age, years & $11.1 \pm 0.4$ & $11.4 \pm 0.5$ & $11.4 \pm 0.5$ & n.s. & n.s. \\
\hline Male infant & $1.7 \pm 0.1$ & $2.1 \pm 0.2$ & $1.5 \pm 0.2$ & \multirow[t]{2}{*}{ n.s. } & \multirow[t]{2}{*}{$<0.01^{\dagger}$} \\
\hline Female infant & $2.1 \pm 0.2$ & $1.5 \pm 0.2^{\mathrm{a}-\mathrm{c}}$ & $1.8 \pm 0.1$ & & \\
\hline Gestation length, days & $167.8 \pm 0.6$ & $169.4 \pm 0.7$ & $167.4 \pm 0.6$ & n.s. & n.s. \\
\hline 6-month weight & $1,398.9 \pm 20.6$ & $1,447.4 \pm 25.6$ & $1,394.0 \pm 21.8^{\mathrm{b}}$ & n.s. & $<0.06$ \\
\hline \multicolumn{6}{|l|}{ BMI at birth } \\
\hline Male infant & $12.9 \pm 0.3$ & $12.9 \pm 0.4$ & $13.2 \pm 0.3$ & \multirow[t]{2}{*}{$<0.03^{*}$} & \multirow[t]{2}{*}{$<0.05^{\dagger}$} \\
\hline Female infant & $12.1 \pm 0.3$ & $13.8 \pm 0.4^{\mathrm{a}}$ & $12.8 \pm 0.3^{\mathrm{b}}$ & & \\
\hline
\end{tabular}

Mean \pm SEM values are shown. Preconception weight was controlled when examining the influence of pregnancy condition and infant sex on pregnancy weight gain. * Significant main effect of pregnancy conditions. ${ }^{\dagger}$ Significant interaction of pregnancy condition and infant sex. ${ }^{\text {a }}$ Significantly different from controls at $\mathrm{p}<0.05$. ${ }^{\mathrm{b}}$ Significantly different from early stress at $\mathrm{p}<0.05$. $^{\mathrm{c}}$ Significantly different from early stress males at $\mathrm{p}<0.05$.

and sex of infant were still present at 6 months of age $[\mathrm{F}(2$, $128)=2.95, p<0.06]$. Female infants from the early stress condition now weighed a mean of $97 \mathrm{~g}$ more than control females and $130 \mathrm{~g}$ more than females from late stress pregnancies (fig. $2 \mathrm{~b}$ ). As expected, there was a main effect for infant sex. Males weighed approximately $29 \mathrm{~g}$ more than females at the time of birth $[\mathrm{F}(1,128)=5.62$, $\mathrm{p}<$ 0.02] and $71 \mathrm{~g}$ more than females at 6 months of age $[\mathrm{F}(1,128)=5.72, \mathrm{p}<0.02]$.

There was an overall main effect of pregnancy condition on infant BMI at birth $[\mathrm{F}(2,128)=3.68, \mathrm{p}<0.03$; table 1]. Post hoc analyses indicated infants exposed to the early disturbance had significantly larger BMIs than control infants $(\mathrm{p}<0.01)$. Additionally, there was an interaction between pregnancy condition and sex, which was driven by the substantially larger BMIs of early stress females $[F(2,128)=3.09, p<0.05]$. These effects were maintained when controlling for gestation length. Differences in BMI were not due to an effect on linear growth; crown-rump length was not significantly affected by maternal disturbance.

There was also a significant interaction between pregnancy condition and infant sex on growth rate $[\mathrm{F}(2$, $128)=3.17, p=0.05]$. Growth rates of early stress females surpassed both undisturbed control and late stress females. Overall, however, males still increased their 
Fig. 3. Temperament and motor maturity ratings from IBAS. a Infants exposed to late gestational disturbance were more reactive and evinced less emotional regulation $(\mathrm{p}<$ $0.05)$. b They also had immature motor responses $(\mathrm{p}<0.03)$ as compared to infants from undisturbed control pregnancies. Mean \pm values are portrayed. $* \mathrm{p}<0.05$, significantly different from control infants.

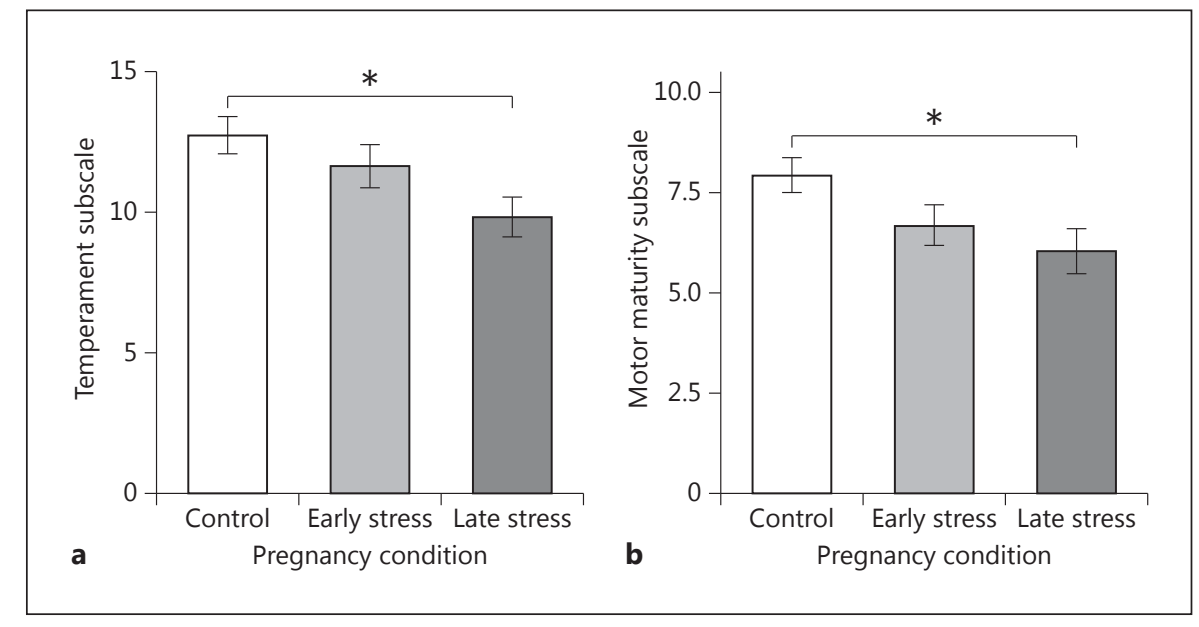

weights at a significantly faster rate than females $[\mathrm{F}(1,128)=4.92, \mathrm{p}<0.03]$.

\section{Infant Behavioral Assessment Scale}

Infants from the three pregnancy conditions differed on the temperament subscale, $[\mathrm{F}(2,116)=4.67, \mathrm{p}<0.01]$, driven primarily by late stress infants scoring a mean 3 points less on emotional control and state regulation than control infants (fig. 3a). Motor maturity also differed significantly across the three pregnancy conditions $[\mathrm{F}(2$, $116)=4.25, \mathrm{p}<0.02$. Post hoc testing indicated late-exposed infants displayed significantly less neuromotor maturity than infants from undisturbed pregnancies (fig. 3b). Neither sensory sensitivity nor orientation revealed an effect of prenatal disturbance. Male and female infants did not differ significantly on any IBAS subscale, and the effects of prenatal disturbance manifested similarly in both sexes.

\section{Discussion}

This study replicates research showing that stress experienced by a pregnant female influences fetal growth and neurobehavioral development $[2,3]$. Even moderate disturbance for just $25 \%$ of the pregnancy impaired motor performance, reduced attention span, and increased emotionality. These findings are consistent with human studies linking maternal anxiety during pregnancy to a more difficult temperament and to attention deficit hyperactivity disorder in older children $[10,16]$. Many have hypothesized these developmental sequelae are mediated in part by the placental transfer of maternal cortisol [13].
Our manipulations did acutely increase maternal cortisol levels. In addition, previous studies using this stress model documented that fetal cortisol secretion may be suppressed in the final weeks of gestation after the disturbance ends, and postnatally the response to dexamethasone is different, indicating a shift in regulatory set points for glucocorticoid feedback $[17,18]$.

The findings on infant growth differ from reports of smaller birth weights [1]. A stunting of growth and reduction in the number of pups is common in rodent experiments, which may reflect the dual influence of more severe stressors and fetal competition for resources in litterbearing species $[12,19]$. In contrast, female infants from the early stress condition had significantly larger birth weights, larger BMIs, and an enhanced growth trajectory sustained through 6 months of age. Three explanations may account for this effect: (1) a compensatory increase in placental size, as found in some nutritionally challenged pregnancies, (2) a metabolic shift in the gravid female similar to the diabetic pregnancy, which also results in larger infants, and (3) a rebound period of accelerated growth, as observed in some small-for-gestational age and premature infants $[1,8,20]$. In keeping with the hypothesis of placental modification, maternal conditions during early development can influence placental vasculature and functioning, including through epigenetic modifications that affect gene transcription [21, 22]. Resolving this question further in primates would require $\mathrm{C}$-section delivery to acquire the placenta, as the mothers typically engage in placentophagia at delivery [23].

Although similar effects on neuromotor maturation and emotionality were found in both sexes and after both stress periods, the enhanced growth was sex-specific and 
observed only after stress early in pregnancy. Male infants are often thought to be more impacted by prenatal insults, and this vulnerability is reflected in the higher prevalence of neurodevelopmental and learning disorders among boys [11,24]. However, a number of papers have reported larger effects on specific measures in female offspring $[8,25]$. Prior studies in primates have yielded inconsistent results: many find no sex differences, while others have detected resilience in the female fetus. In our study, the preconception weight of mothers who bore female infants was slightly heavier $(0.3 \mathrm{~kg})$. Because preconception weight is correlated with infant birth weight, the gravid dams' larger size may have enabled female fetuses to maximize growth during or after the period of early gestational stress. There is also precedent in humans for sex-specific responses to pregravid maternal weight as well as to adiponectin and insulin levels in the third trimester [26]. Other experiments suggest a female fetus may adapt more readily to intrauterine adversity through modifications of placental gene and protein expression $[20,27]$. In humans, the placenta of females is also more responsive to maternal glucocorticoid levels early in gestation [25], consistent with our finding of a sex-specific increase after early stress. One study also indicated exposure to glucocorticoids at critical stages of placental development can increase the efficiency of nutrient transfer to the fetus [20], which when coupled with the possibility of sexually dimorphic placental adaptations, could account for larger female infants despite lower maternal weight gain after early stress.

In conclusion, a delimited period of maternal stress during pregnancy affected neurobehavioral development in infant monkeys, evinced by immature motor reflexes and greater emotionality. These effects were seen in infants born at typical birth weights, as well as in females larger than normal. This enhanced growth for females from the early stress pregnancies resulted in a $10 \%$ difference by delivery, and their faster growth trajectories were maintained through 6 months of age. This study also highlights the importance of distinguishing between moderate gestational stress and chronic challenges that span the entire pregnancy. After a delimited period of early disturbance, there may be compensatory adaptations that promote resilience and even complete recovery.

\section{Acknowledgments}

This research was supported in part by grants from the NICHD and NIMH to C.L.C., which also provided salary for G.R.L. (HD057064, MH104198). D.N.R. received support from the Training Program in Emotion Research (5T32MH018931).

\section{References}

1 Barker DJ, Thornburg KL: Placental programming of chronic diseases, cancer and lifespan: a review. Placenta 2013;34:841-845.

2 Ehrlich DE, Neigh GN, Bourke CH, Nemeth CL, Hazra R, Ryan SJ, Rowson S, Jairam N, Sholar CA, Rainnie DG, Stowe ZN, Owens MJ: Prenatal stress, regardless of concurrent escitalopram treatment, alters behavior and amygdala gene expression of adolescent female rats. Neuropharmacology 2015;97:251258.

3 Schneider ML, Coe CL, Lubach GR: Endocrine activation mimics the adverse effects of prenatal stress on the neuromotor development of the infant primate. Dev Psychobiol 1992;25:427-439.

4 Shah PS, Shah J; Knowledge Synthesis Group on Determinants of Preterm/LBW Births: Maternal exposure to domestic violence and pregnancy and birth outcomes: a systematic review and meta-analyses. J Womens Health (Larchmt) 2010;19:2017-2031.

5 Harville E, Xiong X, Buekens P: Disasters and perinatal health: a systematic review. Obstet Gynecol Surv 2010;65:713-728.
6 Belkacemi L, Nelson DM, Desai M, Ross MG: Maternal undernutrition influences placental-fetal development. Biol Reprod 2010;83: 325-331.

7 Meyer U, Nyffeler M, Yee BK, Knuesel I, Feldon J: Adult brain and behavioral pathological markers of prenatal immune challenge during early/middle and late fetal development in mice. Brain Behav Immun 2008;22: 469-486.

8 Ericsson A, Säljö K, Sjöstrand E, Jansson N, Prasad PD, Powell TL, Jansson T: Brief hyperglycaemia in the early pregnant rat increases fetal weight at term by stimulating placental growth and affecting placental nutrient transport. J Physiol 2007;581:1323-1332.

9 Amugongo SK, Hlusko LJ: Impact of maternal prenatal stress on growth of the offspring. Aging Dis 2014;5:1-16.

10 Kinsella MT, Monk C: Impact of maternal stress, depression and anxiety on fetal neurobehavioral development. Clin Obstet Gynecol 2009;52:425-440.
11 Dipietro JA, Voegtline KM: The gestational foundation of sex differences in development and vulnerability. Neurosci 2015, DOI: 10.1016/j.neuroscience.2015.07.068.

12 Ryan BC, Vandenbergh JG: Intrauterine position effects. Neurosci Biobehav Rev 2002;26: 665-678.

13 Moisiadis VG, Matthews SG: Glucocorticoids and fetal programming part 1: outcomes. Nat Rev Endocrinol 2014;10:403-411.

14 Champoux M, Suomi SJ, Schneider ML: Temperament differences between captive Indian and Chinese-Indian hybrid rhesus macaque neonates. Lab Anim Sci 1994;44:351-357.

15 Coe CL, Lubach GR, Crispen HR, Shirtcliff EA, Schneider ML: Challenges to maternal wellbeing during pregnancy impact temperament, attention, and neuromotor responses in the infant rhesus monkey. Dev Psychobiol 2010;52:625-637.

16 Glover V: Maternal depression, anxiety and stress during pregnancy and child outcome; what needs to be done. Best Pract Res Clin Obstet Gynaecol 2014;28:25-35. 
17 Coe CL, Kramer M, Czéh B, Gould E, Reeves AJ, Kirschbaum C, Fuchs E: Prenatal stress diminishes neurogenesis in the dentate gyrus of juvenile rhesus monkeys. Biol Psychiatry 2003;54:1025-1034.

18 Kapoor A, Lubach GR, Ziegler TE, Coe CL: Hormone levels in neonatal hair reflect prior maternal stress exposure during pregnancy. Psychoneuroendocrinology, in press.

19 Götz AA, Wolf M, Stefanski V: Psychosocial maternal stress during pregnancy: effects on reproduction for F0 and F1 generation laboratory rats. Physiol Behav 2008;93:10551060.
20 Fowden AL, Ward JW, Wooding FPB, Forhead AJ, Constancia M: Programming placental nutrient transport capacity. J Physiol 2006;572:5-15.

21 Myatt L: Placental adaptive responses and fetal programming. J Physiol 2006;572:25-30.

22 van Uitert EM, Exalto N, Burton GJ, Willemsen SP, Koning AHJ, Eilers PHC, Laven JSE, Steegers EAP, Steegers-Theunissen RPM: Human embryonic growth trajectories and associations with fetal growth and birthweight. Hum Reprod 2013;28:1753-1761.

23 Coe CL, Lubach GR: Vital and vulnerable functions of the primate placenta critical for infant health and brain development. Front Neuroendocrinol 2014;35:439-446.

24 Kinney DK, Munir KM, Crowley DJ, Miller AM: Prenatal stress and risk for autism. Neurosci Biobehav Rev 2008;32:1519-1532.
25 Sandman CA, Glynn LM, Davis EP: Is there a viability-vulnerability tradeoff? Sex differences in fetal programming. J Psychosom Res 2013;75:327-335.

26 Simón-Muela I, Näf S, Ballesteros M, Vendrell J, Ceperuelo-Mallafre V, de la Flor M, Megia A: Gender determines the actions of adiponectin multimers on fetal growth and adiposity. Am J Obstet Gynecol 2013;208:481. e1-481.e7.

27 Clifton VL: Review: sex and the human placenta: mediating differential strategies of fetal growth and survival. Placenta 2010; 31(suppl):S33-S39. 